Saudi Journal of Oral and Dental Research

Abbreviated Key Title: Saudi J Oral Dent Res

ISSN 2518-1300 (Print) |ISSN 2518-1297 (Online)

Scholars Middle East Publishers, Dubai, United Arab Emirates

Journal homepage: https://saudijournals.com/sjodr

Review Article

\title{
COVID-19 Pandemic \& Oral Health Care - An Overview of Current Updates
}

Dr. Sudeep C. Bhagvandas ${ }^{1 *}$, Dr. Jubin Hassan Kalathingal ${ }^{2}$, Dr. Waseem Ansar $^{3}$, Dr. Thariq Vengoor Kalathil ${ }^{4}$, Dr. Nikhil Chandran ${ }^{5}$, Dr. Nithin Pratap ${ }^{6}$

${ }^{1}$ Associate Professor \& Head, Department of Public Health Dentistry, Sree Anjaneya Institute of Dental Sciences, Malabar Medical College Campus, Kozhikode, Kerala, India

${ }^{2}$ Specialist Orthodontist, Kings Dental Center, Muaither, Doha, Qatar

${ }^{3}$ Specialist Orthodontist, Hamad Medical Corporation, Doha, Qatar

${ }^{4}$ Specialist Orthodontist, Abeer Medical Center, Batha, Riyadh, KSA

${ }^{5}$ Resident Pediatric Dentist, Department of Dentistry, Government District Hospital, Vadakara, Kerala, India

${ }^{6}$ Assistant Professor, Department of Oral \& Maxillofacial Surgery, Pushpagiri College of Dental Sciences, Thiruvalla, Kerala, India

DOI: $10.36348 /$ sjodr.2020.v05i09.008

| Received: 25.08.2020 | Accepted: 05.09.2020 | Published: 09.09.2020

*Corresponding author: Dr. Sudeep C. B.

\section{Abstract}

The latest threat to global health is the ongoing outbreak of the respiratory disease that was recently given the name Coronavirus Disease 2019 (COVID-19). COVID-19 was recognized in December 2019, caused by a novel coronavirus which is structurally related to the virus that causes severe acute respiratory syndrome (SARS). As in two preceding instances of emergence of coronavirus disease in the past 18 years - SARS and Middle East respiratory syndrome (MERS), the COVID-19 outbreak has posed critical challenges for the public health, research, and medical communities. The goal of an infection control program is to provide a safe working environment for dental health care personnel and their patients. It is crucial for all dental practitioners to be up to date on current Centers for Disease Control and Prevention (CDC) and other important guidelines, equipments, and techniques for proper infection control. Continuous evaluation of infection control practices is important, this ensures the patients and enables the dental healthcare providers confidence that oral health care can be delivered and received in a safe and effective manner.

Keywords: SARS-CoV-2, COVID-19, Coronavirus, Infection Control, Dentistry, Occupational Hazard.

Copyright @ 2020: This is an open-access article distributed under the terms of the Creative Commons Attribution license which permits unrestricted use, distribution, and reproduction in any medium for non-commercial use (NonCommercial, or CC-BY-NC) provided the original author and source are credited.

\section{INTRODUCTION}

Coronavirus (COVID-19) is an enveloped RNA virus that is diversely found in humans and wildlife. A total of six species have been identified to cause disease in humans. They are known to infect the neurological, respiratory, enteric, and hepatic systems. The past few decades have seen endemic outbreaks in the form of Middle East respiratory syndrome coronavirus (MERS-CoV) and severe acute respiratory syndrome related coronavirus (SARS-CoV) [1]. Yet again, we see the emergence of another outbreak due to a new strain called the SARS-CoV-2 virus. The most recent outbreak initially presented as pneumonia of unknown etiology in a cluster of patients in Wuhan, China. The epicenter of infection was linked to seafood and exotic animal wholesale markets in the city. SARS$\mathrm{CoV}-2$ is highly contagious and has resulted in a rapid pandemic of COVID-19. As the number of cases continues to rise, it is clear that these viruses pose a threat to public health [2].
They can be isolated from different animal species. These include birds, livestock, and mammals such as camels, bats, masked palm civets, mice, dogs, and cats. The widespread distribution and infectivity of COV make it an important pathogen [3]. Human pathogenic subtypes of $\mathrm{CoV}$ are associated with mild clinical symptoms. However, severe acute respiratory syndrome related coronavirus (SARS-CoV) and Middle East respiratory syndrome coronavirus (MERS-CoV) are the two notable exceptions. In 2012, MERS-CoV was first detected in Saudia Arabia. It was responsible for 2,494 confirmed cases, which led to 858 fatalities. In 2002, a subtype of the beta-COV rapidly spread across Guangdong, China. This outbreak resulted in 8,000 infections and 774 fatalities in 37 countries [4].

\section{MICROBIOLOGY}

Corona virus is spherical or pleomorphic, single stranded, enveloped RNA and covered with club 
shaped glycoprotein. Corona viruses are four sub types such as alpha, beta, gamma and delta corona virus. Each of sub type corona viruses has many serotypes. Some of them affect human of other affected animals such as pigs, birds, cats, mice and dogs [5, 6].

\section{Mode of Transmission, Symptoms \& Complications: [7-12]}

Transmission of Severe Acute Respiratory Syndrome Corona Virus 2 (SARS-CoV-2) occurs primarily via respiratory droplets from face-to-face contact and, to a lesser degree, via contaminated surfaces. Aerosol spread may occur, but the role of aerosol spread in humans remains unclear. An estimated $48 \%$ to $62 \%$ of transmission may occur via presymptomatic carriers.

Symptoms in hospitalized patients include fever $(70 \%-90 \%)$, dry cough $(60 \%-86 \%)$, shortness of breath $(53 \%-80 \%)$, fatigue $(38 \%)$, myalgias $(15 \%$ $44 \%)$, nausea/vomiting or diarrhea $(15 \%-39 \%)$, headache, weakness (25\%), and rhinorrhea (7\%). Anosmia or ageusia may be the sole presenting symptom in approximately $3 \%$ of individuals with COVID-19.

Laboratory abnormalities among hospitalized patients include lymphopenia (83\%), elevated inflammatory markers (eg, erythrocyte sedimentation rate, C-reactive protein, ferritin, tumor necrosis factor$\alpha$, IL-1, IL-6), and abnormal coagulation parameters (eg, prolonged prothrombin time, thrombocytopenia, elevated D-dimer [46\% of patients], low fibrinogen).

Radiographic findings of individuals with COVID-19 include bilateral, lower-lobe predominate infiltrates on chest radiographic imaging and bilateral, peripheral, lower-lobe ground-glass opacities and/or consolidation on chest computed tomographic imaging.

Complications among hospitalized patients with COVID-19 include pneumonia (75\%); Acute Respiratory Distress Syndrome (15\%); acute liver injury, characterized by elevations in aspartate transaminase, alanine transaminase, and bilirubin $(19 \%)$; cardiac injury, including troponin elevation $(7 \%$ - 17\%), acute heart failure, dysrhythmias, and myocarditis; prothrombotic coagulopathy resulting in venous and arterial thromboembolic events (10\% $25 \%)$; acute kidney injury (9\%); neurologic manifestations, including impaired consciousness (8\%) and acute cerebrovascular disease (3\%); and shock (6\%).Rare complications among critically ill patients with COVID-19 include cytokine storm and Macrophage Activation Syndrome (ie, secondary hemophagocytic lymphohistiocytosis).

\section{Assessment and Diagnosis: [13-16]}

Diagnosis of COVID-19 is typically made using polymerase chain reaction testing via nasal swab.
However, because of false negative test result rates of SARS-CoV-2 PCR testing of nasal swabs, clinical, laboratory, and imaging findings may also be used to make a presumptive diagnosis.

Diagnostic Testing: Polymerase Chain Reaction and Serology Reverse transcription polymerase chain reaction-based SARS CoV-2 RNA detection from respiratory samples (eg, nasopharynx) is the standard for diagnosis. However, the sensitivity of testing varies with timing of testing relative to exposure. One modeling study estimated sensitivity at $33 \% 4$ days after exposure, $62 \%$ on the day of symptom onset, and $80 \% 3$ days after symptom onset. Factors contributing to false-negative test results include the adequacy of the specimen collection technique, time from exposure, and specimen source. Lower respiratory samples, such as bronchoalveolar lavage fluid, are more sensitive than upper respiratory samples. Several serological tests can also aid in the diagnosis and measurement of responses to novel vaccines. However, the presence of antibodies may not confer immunity because not all antibodies produced in response to infection are neutralizing.

Whether and how frequently second infections with SARS-CoV-2 occur remain unknown. Does the presence of antibody changes susceptibility to subsequent infection or how long antibody protection lasts are also unknown. IgM antibodies are detectable within 5days of infection, with higher IgM levels during week 2 to 3 of illness, while an $\mathrm{IgG}$ response is first seen approximately 14 days after symptom onset. Higher antibody titers occur with more severe disease. Available serological assays include point-of-care assays and high throughput enzyme immunoassays. However, test performance, accuracy, and validity are variable.

\section{Treatment \& Prevention: [19-22]}

There is no specific antiviral treatment recommended for COVID-19, and no vaccine is currently available. The treatment is symptomatic, and oxygen therapy represents the first step for addressing respiratory impairment. Non-invasive (NIV) and invasive mechanical ventilation (IMV) may be necessary in cases of respiratory failure refractory to oxygen therapy. Again, intensive care is needed to deal with complicated forms of the disease.

Concerning ARDS treatment, accumulating knowledge on the pathophysiology of lung damage, has gradually induced clinicians to review strategies for dealing with respiratory failure. As Gattinoni et al. suggested, COVID-19-induced ARDS (CARDS) is not a "Typical" ARDS. This aspect of the disease is of fundamental importance and has probably negatively affected the therapeutic approach in the early stages of the pandemic. Indeed, despite at beginning of the pandemic, early IMV was postulated as the better 
strategy for addressing CARDS, in COVID-19 pneumonia the typical ARDS respiratory mechanics featuring reduced lung compliance (i.e., ability to stretch and expand lungs) cannot be found. On the contrary, in CARDS, good pulmonary compliance can be demonstrated. As a consequence, and in contrast to what was initially believed, NIV can have a key role in CARDS therapy.

Other Therapies Include: O2 Fast Challenge, HFNO and Non-invasive Ventilation, Intubation, Protective Mechanical Ventilation, and use of Corticosteroids, Antiviral agents, immunomodulatory drugs, Serotherapy, Anticoagulant, Inflammation inhibitors ect. When the disease results in complex clinical pictures of MOD, organ function support in addition to respiratory support, is mandatory. Extracorporeal membrane oxygenation (ECMO) for patients with refractory hypoxemia despite lungprotective ventilation should merit consideration after a case-by-case analysis. It can be suggested for those with poor results to prone position ventilation. Unselective or inappropriate administration of antibiotics should be avoided, although some centers recommend it.

Preventive measures are the current strategy to limit the spread of cases. Because an epidemic will increase as long as R0 is greater than 1 (COVID-19 is 2.2), control measures must focus on reducing the value to less than 1. Preventive strategies are focused on the isolation of patients and careful infection control, including appropriate measures to be adopted during the diagnosis and the provision of clinical care to an infected patient. For instance, droplet, contact, and airborne precautions should be adopted during specimen collection, and sputum induction should be avoided.

The WHO and other organizations have issued the following general recommendations: [12, 15]

1. Avoid close contact with subjects suffering from acute respiratory infections.

2. Wash your hands frequently, especially after contact with infected people or their environment.

3. Avoid unprotected contact with farm or wild animals.

4. People with symptoms of acute airway infection should keep their distance, cover coughs or sneezes with disposable tissues or clothes and wash their hands.

5. Strengthen, in particular, in emergency medicine departments, the application of strict hygiene measures for the prevention and control of infections.

6. Individuals that are immunocompromised should avoid public gatherings.

The most important strategy is to frequently wash the hands and use portable hand sanitizer and avoid contact with their face and mouth after interacting with a possibly contaminated environment. Isolation and contact tracing alone represent insufficient measures to control the spread of the disease. Nevertheless, their efficacy increases with the distancing. To this regard, a modeling study with data from over 40,000 participants in the UK, demonstrated that the combination of isolation and contact tracing with physical distancing measures can be effective for reducing the accounting of cases that would need to self-isolate and of contacts that would need to be traced, controlling in turn, the disease transmission. Healthcare workers caring for infected individuals should utilize contact and airborne precautions to include PPE such as N95 or FFP3 masks, eye protection, gowns, and gloves to prevent transmission of the pathogen [19, 21, 22]

\section{Oral Health Care Perspective}

Due to the inherent characteristics of dental settings and aerosol involvement, the risk of crossinfection could be high between dental practitioners and patients. Dental practices and hospitals in India, as we have a sizable number of COVID-19 patients and several hotspots, most of the operation has been closed. Only emergency and urgency cases are taken up. Even in such cases, strict and effective infection control protocols are to be followed [23].

\section{Oral manifestations}

A recent survey concluded that more than half of the patients suffered from dysgeusia / amblygeustia. The Centers for Disease Control and Preventions (CDC) included recent loss of taste sensation as an early symptom of COVID-19 (American Centers of Disease Control and Prevention, 2020b). Other oral manifestations reported so far include oral unspecific ulcerations (affecting both keratinized and nonkeratinized epithelium), xerostomia, opportunistic fungal infections, recurrent oral herpes simplex virus-1 infection, fixed drug eruptions, and gingivitis. There is no conclusive evidence whether oral lesions associated with COVID19 are typical of direct viral invasion or occurring as a result of systemic deterioration or following adverse drug reactions. However, the most likely reason could be linked to the fact that oral tissues (salivary glands and tongue) show high degree of ACE2 expression and to the presence of FURIN (an enzyme that facilitates cellular entry of SARS-CoV-2)[24-26].

\section{Saliva and COVID-19 [25 - 28]}

SARS-CoV-2 has been found to be in high concentration in saliva of infected patients which makes it a potential route of transmission. Salivary droplets consist of droplet nuclei of microorganisms in a mixture of moisture, generated by an infected person during coughing, sneezing, talking, or exhalation. The potential risk of transmission through salivary droplets depends on (i) how long the droplets remain in air and (ii) how long the virus remains infectious in the droplet. 
The potential diagnostic value of saliva in detecting COVID-19 virus is still under research. Throat swabs are exclusively used for diagnostic confirmation of COVID-19 infection. However, throat swabs are relatively invasive, induce cough and bleeding, increasing risk of healthcare workers to this infection. Saliva stands at the entry of respiratory system. Moreover, collection of saliva is less invasive, more acceptable to patients and less hazardous to health care workers. Saliva can be collected using any of the following three approaches: coughing out (deep throat saliva), salivary swabs, and directly from salivary gland duct. A recent study concluded that deep throat saliva has the highest rate of positive virus detection.

\section{Recommendations for Providing Dental Care during COVID-19 Pandemic: [29-41]}

\section{Dental clinic modification}

a. Reception/Waiting area

Display visual alerts at the entrance of the clinic and reception area about respiratory hygiene, cough etiquette, social distancing, and disposal of contaminated items in trash cans. As soon as the patient enters the reception area, ask them to wash their hands using hand wash or alcohol-based hand rub. Use tissue paper or hand dryer to dry the hands instead of towels. Tissue paper dispenser and foot-operated waste bin are mandatory. Include temperature recordings as part of your routine patient assessment before performing any dental procedure. A noncontact forehead thermometer can be used to measure the patient's body temperature. Patients presenting with fever or respiratory disease/symptoms should be registered and referred to designated hospitals.

\section{Include a screening questionnaire with questions}

1. Any history of fever/respiratory illness, including cough or difficulty in breathing in the last 14 days?

2. Any history of contact by you or any household member with a known COVID-19 patient in the past 14 days?

3. Any history of international travel by you or any household member or to areas of suspected community spread in the last 14 days?

Maintain social distancing in the reception area by placing chairs, preferably $1 \mathrm{~m}$ apart. Instruct the patient to wear a mask while waiting in the reception area and maintain respiratory hygiene by covering their mouth and nose during coughing and sneezing.

Remove magazines, articles, toys, and other objects from the reception area that may be touched by others and are difficult to disinfect. Install glass/plastic barrier at the reception desk. Cashless/contactless payment methods should be encouraged. Avoid usage of commercial split/centralized/window air conditioners unless equipped with high-efficiency particulate air
(HEPA) filters. It is recommended to use natural and mechanical ventilation using fans and exhaust.

\section{b. Operatory Area}

Installation of high vacuum extra oral suction devices recommended. Maintain natural air circulation within the operatory, through frequent opening of windows and by using an exhaust blower to extract the room air into the atmosphere. Place a table fan behind the operator and let the air flow toward the patient. A strong exhaust fan is recommended to create a unidirectional flow of air away from the patient. Avoid the use of a ceiling fan while performing procedure. The window air condition system/split AC should be frequently serviced, and filters cleaned. Commercially available electrostatic air conditioner filters can be used. Use of indoor portable air cleaning system equipped with HEPA filter and UV light may be used.

\section{c. Changing Room}

Changing room to be available for staff and all workers. Dedicated area for donning and doffing of personal protective equipment (PPE).

\section{Protocols for Dental Patient Management: a. Tele-screening and Triaging}

The objective of triaging is to facilitate the scheduling of patients based on the level of need. Triaging helps to limit incoming patients while prioritizing emergency care. Thus, there are three categories of patients requiring 1) emergency, 2) urgent, and 3) scheduled/elective care.

\section{b. Dental Health Care Professional Guidelines}

Strict adherence to hand hygiene protocols should be followed. The highest level of PPE, i.e., gloves, gown, goggles, face shields, and an N95 or higher-level respirator must be used during emergency dental care. N95 masks by the national institute for occupational safety and health / FFP2 masks (filter face piece) set by the European Union are recommended. If available FFP3 (N99) standard mask should be used and, in COVID-19 positive patients, this would be considered essential. Recommendation of PPE for dental staff is provided in.

\section{c. Pre-procedural Modifications}

Drape the patient preferably with single-use, disposable plastic apron. Ask the patient to remove the mask. Provide pre-procedural mouth rinse, this helps in effective reduction of salivary microbial load which can be achieved by rinsing with $0.2 \%$ povidone-iodine or $1 \%$ hydrogen peroxide before the procedure. Some studies carried out has concluded that chlorhexidine is ineffective against COVID-19.

\section{d. Procedural Modifications}

According to guidelines given by Ministry of Health and Family Welfare dated 19 May 2020, only emergency and urgent procedures to be carried out and 
all routine and elective dental procedures should be deferred for a later review until new policy/guidelines are issued. Practice modifications for emergency, urgent care, and specialty wise modification to be adopted as and when regular services resume.

\section{e. Emergency Treatment Protocol for the Management of High-risk Patients}

The Centers for Disease Control and Prevention (CDC) as on 8th April 2020 recommends to postpone all elective procedures, surgeries, and nonurgent dental visits, while prioritizing urgent, emergency visits and procedures now and for the coming several weeks.

Emergency dental treatment for a confirmed/suspected COVID-19 patient if warranted medically, it should only be provided in a hospital or dental setup with adequate airborne precautions (negative pressure or AIIR and an N95 mask). Emergency dental care for non-COVID-19 patients may be provided using appropriate engineering controls, work practices, and infection control protocols. Dentists providing treatment must exercise clinical judgment based on their skill, expertise, unique patient-specific factors while following the protocols issued by the concerned regional dental and health advisories.

\section{f. Management of Resolved COVID-19 Patients}

The emergency dental care for resolved COVID-19 patients is decided using two strategies: a nontest-based strategy and a test-based-strategy.

Nontest-Based-Strategy: At least 3 days (72 h) have passed since recovery (resolution of fever without the use of fever-reducing medications and improvement in respiratory symptoms such as cough or shortness of breath) and at least 7 days have passed since symptoms first occurred.

Test-Based-Strategy: Symptomatic COVID-19 patients: Resolution of fever without the use of feverreducing medications and improvement in respiratory symptoms (e.g., cough, shortness of breath) and negative results from at least two consecutive nasopharyngeal swab specimens collected $\geq 24 \mathrm{~h}$ apart.

Asymptomatic laboratory-confirmed COVID19 patients: At least 7 days have passed since the date of the first positive COVID-19 diagnostic test and have had no subsequent illness.

\section{g. Patient Discharge Protocol}

After the procedure is complete, patient drape has to be removed by the assistant. Patient should be advised to perform hand hygiene. Guide the patient out of the clinic toward reception area. Recording of the prescription and follow up instructions should only be done after doffing the PPE.

\section{Disinfection of the Clinic Settings \\ a. Floor}

Mop the floor with $1 \%$ sodium hypochlorite solution with a contact time of $10 \mathrm{~min}$. Use separate mops for the clinical area. Unidirectional mopping technique should be followed by mopping from inner to outer area. Ideally the floor should be cleaned after every patient or after a major splash or two hourly period. Wash and disinfect the mop with clean water and $1 \%$ sodium hypochlorite and leave it for sundrying.

\section{b. Rest of the Surfaces}

Freshly prepared 1\% sodium hypochlorite (contact time: $10 \mathrm{~min}$ ) is used. Disinfection should be done daily before starting work, after every procedure, and at the end of the day.

\section{c. Delicate Electronic Equipment}

All Electronic equipment should be wiped with alcohol-based rub / spirit $(60 \%-90 \%$ alcohol) swab before each patient contact.

\section{d. Fogging}

This method is called "No-touch surface disinfection." $20 \%$ (w/v) working solution of hydrogen peroxide (stabilized by $0.01 \%$ of silver nitrate) is prepared. The amount of solution required is approximately $1000 \mathrm{~mL}$ per 1000 cubic feet. Immediately after the procedure, exit the room and close the operatory for half hour. This allows the aerosols/droplets to settle down. A 2-step surface cleaning should be performed.

Fogging is done for $45 \mathrm{~min}$ followed by a dwell time of $1 \mathrm{~h}$. The room can then be opened and fans switched on for aeration. Wet surfaces can be dried / cleaned using a sterile cloth or clean cloth.

\section{Waste Management}

The infectious medical and domestic waste of suspected or confirmed COVID-19 patients should be disposed of in double-layered yellow color bags with gooseneck ligation. The bags should be marked and disposed of in accordance with the Biomedical Waste Management and Handling Rules, 2018.

\section{CONCLUSION}

There are several major gaps in our current knowledge of the origin, epidemiology, duration of human transmission, and clinical spectrum of disease regarding SARS CoV-2, information provided here is based on current evidence, but may be modified as more information becomes available [42]. As on $1^{\text {st }}$ August 2020, more than 20 million people worldwide have been infected with SARS-CoV-2. Many aspects of transmission, infection, and treatment remain unclear. Advances in prevention and effective management of COVID-19 will require basic and clinical investigation and public health and clinical interventions [43]. 
Health-care workers caring for patients with suspected or confirmed infection, or handling specimens from them, should practice standard operating procedure and updated infection control precautions at all times. Cross contamination can place the dentist at serious risk. Practices and policies for extending and expanding oral health care must be based on ethical and safety concerns by persons delivering oral prevention, treatment, and care to patients [44].

Hence, it becomes imperative for dentists to realize that the solution to this risk lies within us. Adopting and practicing the latest standard infection control guidelines in routine dental practice and also prevention and taking the necessary precautions is the basic requirement that can help keep the menace of cross contamination and infection away.

\section{REFERENCES}

1. Zhu, N., Zhang, D., Wang, W., Li, X., Yang, B., Song, J., \& Niu, P. (2020). China Novel Coronavirus Investigating and Research Team. A novel coronavirus from patients with pneumonia in China, 2019. N Engl J Med, 382(8), 727-733.

2. Zhong, N. S., Zheng, B. J., Li, Y. M., Poon, L. L. M., Xie, Z. H., Chan, K. H., ... \& Liu, X. Q. (2003). Epidemiology and cause of severe acute respiratory syndrome (SARS) in Guangdong, People's Republic of China, in February, 2003. The Lancet, 362(9393), 1353-1358.

3. Zaki, A. M., Van Boheemen, S., Bestebroer, T. M., Osterhaus, A. D., \& Fouchier, R. A. (2012). Isolation of a novel coronavirus from a man with pneumonia in Saudi Arabia. New England Journal of Medicine, 367(19), 1814-1820.

4. Goldsmith, C. S., Tatti, K. M., Ksiazek, T. G., Rollin, P. E., Comer, J. A., Lee, W. W., ... \& Zaki, S. R. (2004). Ultrastructural characterization of SARS coronavirus. Emerging infectious diseases, 10(2), 320.

5. Lu, R., Zhao, X., Li, J., Niu, P., Yang, B., Wu, H., \& Bi, Y. (2020). Genomic characterisation and epidemiology of 2019 novel coronavirus: implications for virus origins and receptor binding. The Lancet, 395(10224), 565-574.

6. Lam, T. T. Y., Jia, N., Zhang, Y. W., Shum, M. H. H., Jiang, J. F., Zhu, H. C., \& Li, W. J. (2020). Identifying SARS-CoV-2-related coronaviruses in Malayan pangolins. Nature, 1-4.

7. Hoffmann, M., Kleine-Weber, H., Schroeder, S., Krüger, N., Herrler, T., Erichsen, S., \& Müller, M. A. (2020). SARS-CoV-2 cell entry depends on ACE2 and TMPRSS2 and is blocked by a clinically proven protease inhibitor. Cell.

8. Sungnak, W., Huang, N., Bécavin, C., Berg, M., Queen, R., Litvinukova, M., \& Worlock, K. B. (2020). SARS-CoV-2 entry factors are highly expressed in nasal epithelial cells together with innate immune genes. Nature medicine, 26(5), 681687.
9. Ramadan, N., \& Shaib, H. (2019). Middle East respiratory syndrome coronavirus (MERS-CoV): A review. Germs, 9(1), 35.

10. Jonas, O., \& Seifman, R. (2019). Do we need a Global Virome Project?. The Lancet Global Health, 7(10), e1314-e1316.

11. Enserink, M. (2020). Update:'A bit chaotic.'Christening of new coronavirus and its disease name create confusion. Science.

12. World Health Organization. (2020). WHO Director-General's remarks at the media briefing on 2019-nCoV on 11 February 2020. World Health Organization, Geneva. Available via https://www. who. int/dg/speeches/detail/who-director-generals-remarks-at-the-media-briefing-on-2019-ncov-on11-february-2020. Accessed, 10.

13. Gorbalenya, A. E., Baker, S. C., Baric, R., Groot, R. J. D., Drosten, C., Gulyaeva, A. A., ... \& Penzar, D. (2020). Severe acute respiratory syndromerelated coronavirus: The species and its viruses-a statement of the Coronavirus Study Group.

14. World Health Organization. (2005). Statement on the Second Meeting of the International Health Regulations. Emergency Committee regarding the outbreak of novel coronavirus (2019-nCoV); 2005. Available from: https://www.who.int/newsroom/detail/30-01-2020-statement-on-thesecondmeeting-of-the-international-healthregulations-(2005)-emergency-committeeregarding-the-outbreak-of-novelcoronavirus-(2019ncov), accessed on July 27, 2020.

15. Meng, L., Hua, F., \& Bian, Z. (2020). Coronavirus disease 2019 (COVID-19): emerging and future challenges for dental and oral medicine. Journal of Dental Research, 99(5), 481-487.

16. Phelan, A. L., Katz, R., \& Gostin, L. O. (2020). The novel coronavirus originating in Wuhan, China: challenges for global health governance. Jama, 323(8), 709-710.

17. Alharbi, A., Alharbi, S., \& Alqaidi, S. (2020). Guidelines for dental care provision during the COVID-19 pandemic. The Saudi Dental Journal.

18. Ather, A., Patel, B., Ruparel, N. B., Diogenes, A., \& Hargreaves, K. M. (2020). Coronavirus disease 19 (COVID-19): implications for clinical dental care. Journal of endodontics.

19. CDA. (2020). Practice interruption due to COVID19 (coronavirus). 2020. Accessed on July 20th 2020.

20. Carroll, D., Daszak, P., Wolfe, N. D., Gao, G. F., Morel, C. M., Morzaria, S., ... \& Mazet, J. A. (2018). The global virome project. Science, 359(6378), 872-874.

21. Carroll, D., Watson, B., Togami, E., Daszak, P., Mazet, J. A., Chrisman, C. J., ... \& Burci, G. L. (2018). Building a global atlas of zoonotic viruses. Bulletin of the World Health Organization, 96(4), 292.

22. Centres for Disease Control and Prevention. (2013). Prevention strategies for seasonal influenza 
Sudeep C. B et al., Saudi J Oral Dent Res, September, 2020; 5(9): 456-462

in healthcare settings. Guidelines and Recommendations.

23. Bhatnagar, P. (2020). What is the 'COVID-ified' future for dentists? 2020. Accessed on August 2nd 2020.

24. Bharadwaj, S. (2020). There is an urgent need to resuscitate dentistry: Dr. Srivats Bharadwaj. 2020. Accessed on August 6th 2020.

25. Ahmed, M. A., Jouhar, R., Ahmed, N., Adnan, S., Aftab, M., Zafar, M. S., \& Khurshid, Z. (2020). Fear and practice modifications among dentists to combat Novel Coronavirus Disease (COVID-19) outbreak. International journal of environmental research and public health, 17(8), 2821.

26. Chen, L., Zhao, J., Peng, J., Li, X., Deng, X., Geng, Z., ... \& Wang, L. (2020). Detection of 2019-nCoV in saliva and characterization of oral symptoms in COVID-19 patients. Available at SSRN 3556665.

27. Segura- Egea, J. J., Gould, K., Şen, B. H., Jonasson, P., Cotti, E., Mazzoni, A., ... \& Dummer, P. M. H. (2018). European Society of Endodontology position statement: the use of antibiotics in endodontics. International Endodontic Journal, 51(1), 20-25.

28. Centers for Disease Control and Prevention. (2020). Dental Settings. Interim Infection Prevention and Control Guidance for Dental Settings During the COVID-19 Response.

29. CDC Guidance for Providing Dental Care during COVID- 19.

https://www.cdc.gov/oralhealth/infectioncontrol/sta tement- COVID.html. Accessed on 2020 July 30.

30. Interim Guidance for the Use of Masks to Control Seasonal Influenza Virus Transmission Guidelines and Recommendations. (2020). Available from: https://www.cdc.gov/flu/professionals/infectioncon trol/maskguidance.htm. Accessed on 2020 July 30.

31. Kariwa, H., Fujii, N., \& Takashima, I. (2006). Inactivation of SARS coronavirus by means of povidone-iodine, physical conditions and chemical reagents. Dermatology, 212(Suppl. 1), 119-123.

32. Samaranayake, L. P., \& Peiris, M. (2004). Severe acute respiratory syndrome and dentistry: a retrospective view. The Journal of the American Dental Association, 135(9), 1292-1302.

33. Jain, K. V., Simon, S. P., Jain, J., Supreetha, S., Mathew, M. J., \& Sudeep, C. B. (2013). Comparative antibacterial effectiveness of alcohol and herbal based commercially available hand antiseptics. Journal of Indian Association of Public Health Dentistry, 11(2), 27.

34. Sudeep, C. B., Joseph, J., Chaitra, T., Joselin, J., Nithin, P., \& Jose, J. (2017). KAP study to assess biomedical waste management in a dental college in south India. World J Pharm Pharm Sci, 6, 17881794.

35. CB, S., Chandran, N., Jacob, J., \& Johny, J. (2019). Scholars Journal of Applied Medical Sciences.

36. Xiao, Y., Tang, B., Wu, J., Cheke, R. A., \& Tang, S. (2020). Linking key intervention timing to rapid decline of the COVID-19 effective reproductive number to quantify lessons from mainland China. International Journal of Infectious Diseases, 97, 296-298.

37. Le, T. T., Andreadakis, Z., Kumar, A., Roman, R. G., Tollefsen, S., Saville, M., \& Mayhew, S. (2020). The COVID-19 vaccine development landscape. Nat Rev Drug Discov, 19(5), 305-306.

38. Lurie, N., Saville, M., Hatchett, R., \& Halton, J. (2020). Developing Covid-19 vaccines at pandemic speed. New England Journal of Medicine, 382(21), 1969-1973.

39. A guidance handbook of Indian dental association kerala state: Guidelines and recommendations for dental practitioners and auxiliaries. (2020). Available

from: https://www.idakerala.com/downloads/idaksb_covi dguidelines_ver_1.0.pdf. Accessed on 2020 July 24.

40. Management of acute dental problems during COVID- 19 pandemic. (2020). Available from: http://www.sdcep.org.uk/wp- content/uploads/202 0/03/SDCEP- MADP- COVID- 19- guide- 3003 20.pdf. Accessed on 2020 July 30.

41. List, N. (2020). Disinfectants for use against SARS- CoV-2. 2020. Available from: https://www.epa.gov/pesticide- registration/list- $n$ - disinfectants- ${ }^{-}$use- against- ${ }^{-}$sars $^{-}$cov $^{-} 2$. Accessed on 2020 July 30.

42. Prevention Strategies for Seasonal Influenza in Healthcare Settings Guidelines and Recommendations. (2020). Available from: https://www.cdc.gov/flu/professionals/ infectioncontrol/healthcaresettings.htm. Accessed on 2020 July 30.

43. Kariwa, H., Fujii, N., \& Takashima, I. (2006). Inactivation of SARS coronavirus by means of povidone-iodine, physical conditions and chemical reagents. Dermatology, 212(Suppl. 1), 119-123.

44. Qudeimat, M. A., Farrah, R. Y., \& Owais, A. I. (2006). Infection control knowledge and practices among dentists and dental nurses at a Jordanian university teaching center. American journal of infection control, 34(4), 218-222. 